\author{
Military Technical College \\ Kobry El-Kobbah, \\ Cairo, Egypt
}

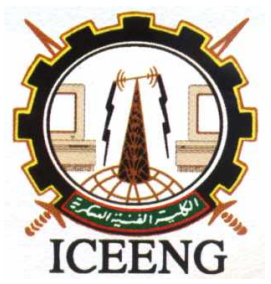

\title{
Transmission cost allocation schemes in competitive power systems
} By

\author{
Adel Ali Abou El Ela* Ragab Abdel aziz El-Sehiemy**
}

\section{$\underline{\text { Abstract: }}$}

This paper presents different suggested transmission cost allocation (TCA) schemes for distributing the transmission usage costs to the system individuals. Different independent system operator (ISO) visions are presented using the proportional rata and flow-based TCA methods. There are two flow-based TCA schemes (FTCA). The first FTCA generalizes the equivalent bilateral exchanges (EBE) concepts for lossy networks through two-stage procedure. The second FTCA approach is based on the modified sensitivity factors (MSF). These factors are developed from the actual measurements of power flows in transmission lines and the injected power at different buses. The proposed schemes exhibit desirable apportioning properties and are easy to implement and understand. Case studies based on two test systems, which are: 5-bus and IEEE 14bus test systems, are carried out to show the capability of the proposed schemes for solving the TCA problem.

\section{Keywords:}

Competitive power markets, equivalent bilateral exchanges, sensitivity analysis, transmission cost allocation.

* Faculty of Engineering, Minoufiya University, Shebin El Kom, Egypt

** The Arab Contractor Osman Ahmed Osman \& Co., Egypt 


\section{Introduction:}

The transmission cost allocation (TCA) to system individuals is one goal of deregulated power systems operator. The problem is how to allocate the total cost of transmission between all the users in an equitable way which provides them with the correct allocation level based on economical signals. The TCA concepts are more general than the wheeling concept which is the transfer of transacted power between two or more utilities through a transmission network of the third one. The cost of transmission usage and the methodology, by which it is computed, is a high priority problem throughout the power industry due to the growth in transmission facilities, the cost differentials between utility companies, and the dramatic growth in non-utility generation capacity. The allocation problems are those associated with determination of generators' contribution to supply of concrete loads, power flows from each generator by the network equivalent circuit and power transmission losses. In real time operation, generators and consumers engage in power transactions. Commonly agreed features of TCA methods are to provide locational signals and incentives to encourage efficient use of the transmission facilities. They also must comply with some conditions to avoid cross-subsidies and to be transparent and easy to implement, to ensure cost recovery, to provide adequate economic signals and to have continuity with time.

Consumer meters measure their actual consumption, while generator meters measure their actual production level. The importance of the transmission system in the new deregulated environment was emphasized as a facilitator of generator competition, allowing generators to allocate their production in consumer centers and enabling consumers to benefit from that competitive environment. Within that framework, the transmission tariff system and the usage cost allocation must preserve an adequate resource allocation among market agents. It is desired that transmission prices and payment do not disturb decisions for new-generation investment, for generator operation, and for consumer demand.

The TCA methods presented in literature could be classified as embedded TCA methods and marginal methods [1]. Marginal cost methods do not guarantee cost recovering in real networks. Embedded cost methods allocate the transmission costs according to the extent of use of generators and consumers. Several methods based on the embedded cost have been presented for different systems. They can be divided into rolled-in methods and load based methods. Rolled-in methods charge a fixed amount per energy unit, and their main drawbacks are that they ignore actual network use and that they do not send adequate economic signals to grid users. Flow-based methods charge the users in proportion to their use the of grid facilities. The flow-based methods classified as proportional or differential methods. The advantages of proportional method are: it is simple to understand and provides grid use and load sharing among generators. Differential methods are well known in literature and are based on the sensitivities of 
branch flows to power injection in nodes. These sensitivities depend on the choice of the slack bus in the studied case therefore; there is a part of arbitrariness in the allocation. A flow based method reported in [2] used the so-called EBE. To build the EBE, each demand is proportionally assigned a fraction of each generation, and conversely. Each generation is proportionally assigned a fraction of each demand, in such a way as both Kirchhoff's laws, are satisfied. Reference [3] presented two procedures based on the Zimpedance matrix and the injected powers. Both procedures to allocate the cost of the transmission network to generators and demands are based on circuit theory. Reference [4] presented a method that integrates cooperation and coordination among the agents and their physical and economic use of the network to allocate charges among users of a transmission system. In particular, cooperative game theory arises as a most convenient tool to solve cost allocation problems [5]. The cost of transmission system usage was presented based on an economic measure of power markets in [6]. In [7], the cooperative game theory based procedure was presented for electricity tracing. A variety of applications in both planning and operation require repetitive computation of power flow and power losses in transmission lines. Sensitivity factors play a key role in many system security analysis and market applications. In [8], the contribution of individual generators to loads and flows was discussed. In [9], a topological sensitivity distribution factors for both of generation and load for supplement charge allocation in transmission open access were found. The modified topological distribution factors were presented in [10] to consider the effects of transmission losses as separate nodes. The generalized generation distribution factors (GGDF) was used for obtaining the power flows in transmission lines in terms of the injected power generations [11].

The main contribution of this paper is to propose modified versions of TCA schemes. Some of the proposed schemes are based on proportional rata concept and other schemes are based on the modified EBE (MEBE) principles and the modified sensitivity factors (MSF).

\section{$\underline{\text { 2. Modified Sensitivity Factors }}$}

The proposed MSF are introduced depend on the actual power system measurements for power flows in the transmission lines and the corresponding injected power at different buses, $P I$, as:

$$
\begin{aligned}
& P F=D_{m} . P I \\
& \mathrm{PF}=\left[\mathrm{PF}_{1} \mathrm{PF}_{2} \mathrm{PF}_{3} \ldots ., \mathrm{PF}_{\mathrm{k}}, \ldots ., \mathrm{PF}_{\mathrm{NL}}\right], \mathrm{k}=1,2, \ldots ., \mathrm{NL} \\
& \mathrm{PI}=\left[\mathrm{PI}_{1} \mathrm{PI}_{2} \mathrm{PI}_{3} \ldots . ., \mathrm{PI}_{\mathrm{i}}, \ldots \ldots \ldots, \mathrm{PI}_{\mathrm{NB}}\right], \mathrm{i}=1,2, \ldots \ldots, \mathrm{NB} \\
& P I_{i}=P G_{i}-P D_{i}
\end{aligned}
$$


Where:

$\mathrm{D}_{\mathrm{m}}$ is the proposed MSF, NL is the number of transmission lines and NB is the number of system buses. The initial power flows $\left(P F^{0}\right)$ in terms of initial injected powers $\left(P I^{0}\right)$ can be expressed as:

$P F^{0}=D_{m} \cdot P I^{0}$

By multiplying both sides of Equation (3) by $\left(\left(P I^{0}\right)^{t} \cdot\left(\left(P I^{0}\right) \cdot\left(P I^{0}\right)^{t}\right)^{(-1)}\right)$, it can be obtained:

$$
\begin{aligned}
& P F^{0} \cdot\left(P I^{0}\right)^{t} \cdot\left(\left(P I^{0}\right) \cdot\left(P I^{0}\right)^{t}\right)^{(-1)}= \\
& D_{m} \cdot\left(P I^{0}\right) \cdot\left(P I^{0}\right)^{t} \cdot\left(\left(P I^{0}\right) \cdot\left(P I^{0}\right)^{t}\right)^{(-1)}=D_{m} \\
& \text { or } D_{m}=P F^{0} \cdot\left(P I^{0}\right)^{t} \cdot\left(\left(P I^{0}\right) \cdot\left(P I^{0}\right)^{t}\right)^{(-1)}
\end{aligned}
$$

From Equation (5), it can be founded that the proposed MSF are dependent on the actual initial measurements of the power flows in transmission lines and the injected power at different buses. The power flow in transmission line $\mathrm{k}\left(P F_{k}\right)$ can be expressed as:

$$
\begin{aligned}
& P F_{k}=\sum_{i=1}^{N B}\left(\left(D_{m}\right)_{k, i} \cdot P I_{i}\right) \\
& \left(D_{m}\right)_{k, i}=P F_{k}^{0} \cdot\left(P I_{k, i}^{0}\right)^{t} \cdot\left(\left(P I_{k, i}^{0}\right) \cdot\left(P I_{k, i}^{0}\right)^{t}\right)^{(-1)}
\end{aligned}
$$

$\left(D_{m}\right)_{k, i}$ is the MSF between line $\mathrm{k}$ and generation $\mathrm{i}$, and $\left(P \mathrm{I}_{k, i}^{0}\right)^{t}$ denotes a transposed vector of power generation vector $P I_{k, i}^{0}$. The advantage of the proposed MSF is that all system buses are considered in the calculation of the power flows.

\section{Suggested transmission cost allocation Schemes:}

Different proportional-based schemes are suggested to allocate the transmission losses for network users [12]. This paper generalizes the loss allocation options for TCA problems. The cost allocated at each bus is computed using the TCA allocation vector $S_{i}$ as:

$C_{a, k}^{i}=S_{i} \cdot C_{k}$

Where,

$C_{a, k}^{i}$ is the cost allocated at bus $i$ to usage of line $k$,

$S_{i}$ is the allocation factor at bus $i$.

$C_{k}$ is the total cost of line $k$.

The suggested TCA allocation schemes are:

\section{Scheme 1: Current injected allocation based}

This scheme is based on the injected current $\left(I_{i}\right)$ at bus $i$. However, the $S_{i}$ vector is 
computed as a percentage of the total injected current at $N B$ - buses, as:

$S_{i}=I_{i} / \sum_{i=1}^{N B} I_{i}$

\section{Scheme 2: Power demand based}

This scheme is suggested based on the power demand at bus $j,\left(P D_{j}\right)$. However, the vector $S_{j}$ is computed as a percentage of the total power demand at $N D$-buses, as:

$$
S_{j}=P D_{j} / \sum_{j=1}^{N D} P D_{j}
$$

\section{Scheme 3: Maximum bus power used}

This scheme is suggested based on the maximum bus power used of $P D_{i} / P G_{i}$. However, the vector $S_{i}$ is computed as a percentage of the maximum bus power used each bus to the total maximum power used at all buses as:

$$
S_{i}=\operatorname{Max} .\left(P G_{i}, P D_{i}\right) / \sum_{i=1}^{N B} \operatorname{Max} .\left(P G_{i}, P D_{i}\right)
$$

\section{Scheme 4: Combined bus power used}

This scheme is suggested based on the combined bus power used. In this scheme, the vector $S_{i}$ is computed as a percentage of the summation of power generation and load demand at certain bus related to their summation power used at all buses as:

$$
S_{i}=\left(P G_{i}+P D_{i}\right) / \sum_{i=1}^{N B}\left(P G_{i}+P D_{i}\right)
$$

\section{Scheme 5: ISO comparable based}

In this scheme, the suggested allocation process is considered by the ISO as an intermediate vision of the average contribution for each power generations and/or load demand related to the total power generation and/or the total power demand. However, the vector $S_{i}$ can be computed as:

$$
S_{i}=A \operatorname{ver}\left(\left(P G_{i} / \sum_{i=1}^{N B} P G_{i}\right) \text { and } / \text { or }\left(P D_{i} / \sum_{i=1}^{N B} P D_{i}\right)\right)
$$

\section{Scheme 6: Interested participant allocation}

This suggested scheme is based on the network configuration and the net injected bus power. This procedure divides the power usage of each line into two components. The first component is for the sending side $(s)$ and the second one for the receiving side $(r)$. The allocation usage factors are computed as a percentage of the net injected power to the summation of the injected power at both sides of each line. The bus usage components due to line $\mathrm{k}\left(U_{k, s r}\right)$ can be computed as:

$$
P_{k, s}=\left(\left|P I_{s}\right| /\left(|P I|_{s}+\left|P I_{r}\right|\right)\right) U_{k, s r}
$$


$P_{k, r}=\left(\left|P I_{r}\right| /\left(|P I|_{s}+\left|P I_{r}\right|\right)\right) U_{k, s r}$

Then, the TCA allocated at receiving bus (r), due to a number of NR lines connected to bus $r$, is computed as:

$$
\mathrm{P}_{a r}=\sum_{s=1}^{N R}\left(\left|P I_{r}\right| /\left(\left|P I_{s}\right|+\left|P I_{r}\right|\right)\right) U_{k, r s}
$$

Similarly, the TCA at sending bus (s), due to a number of NS lines connected to bus s, is computed as:

$$
P_{a s}=\sum_{r=1}^{N S}\left(\left|P I_{s}\right| /\left(\left|P I_{s}\right|+\left|P I_{r}\right|\right)\right) U_{k, s r}
$$

\section{Scheme 7: Modified equivalent bilateral exchanges (MEBE)}

a) Basics of EBE

The EBE between the power generation at bus $i$ and load demand bus at bus $j$ was defined as [2]:

$$
G D_{i j}=\frac{P G_{i} \cdot P D_{j}}{P_{d}^{s y s}}
$$

Where, in lossless network, the system load demand is:

$$
P_{d}^{s y s}=\sum_{j} P D_{j}=\sum_{i} P G_{i}
$$

The equivalent exchanges $G D_{i j}$ can be viewed as the fraction of generation $\left(P G_{i}\right)$ that supplies the bus load demand $\mathrm{P}_{\mathrm{dj}}$ or equivalent to fraction of power demand $\left(P D_{j}\right)$ supplied by the power generation $\left(P G_{i}\right)$. It is decomposed each individual generation and demand level into linear combination of the EBE. It is straightforward to decompose each individual power generation and consumer into a linear combination of the EBE as:

$$
\begin{aligned}
& P G_{i}=\sum_{j} G D_{i j} \\
& P D_{j}=\sum_{i} G D_{i j}
\end{aligned}
$$

The power flow in line $\mathrm{k}\left(P F_{k}\right)$ is expressed in terms of EBE as:

$$
P F_{k}=\sum_{i, j}\left(\gamma_{k i j} \cdot G D_{i j}\right)
$$

The coefficients $\gamma_{k i j}$ are the generation distribution factors (GDF) which computed under DC load flow. These parameters describe the sensitivity of power flow in line $\mathrm{k}\left(P F_{k}\right)$, with respect to the EBE between the power generation at bus $i$ and load demand at bus $j$. The properties of the presented EBE in [2] were:

i) Bilateral exchanges between generators and demand at the same bus $\mathrm{m}$ do not 
make use of the network.

ii) Every power generators and load demand contribute a positive amount to the combined network use.

iii) The rate of line used remains stable for different operating conditions.

b) Proposed MEBE-based allocation procedure

In this paper, the EBE is modified for the lossy network using two-stage procedure. In the first stage, the transmission losses are allocated to system users and modify the actual power generation or load demand levels at different buses. The power generation $\left(P G_{i}\right)$ and load demand $\left(P D_{j}\right)$ are replaced by their virtual power generation levels at generation buses $\left(P G_{i}^{v}\right)$ or virtual power demand at demand buses $\left(P D_{j}^{v}\right)$.

At the generation buses side, the power loss allocated component $\left(P_{i}^{\mathrm{lg}}\right)$ is subtracted from the actual power generation level.

Then, $P G_{i}^{v}$, at bus i, is computed as:

$P G_{i}^{\mathrm{v}}=P G_{i}-P_{i}^{\mathrm{lg}}$

At demand buses side, the loss allocated component to demand buses $\left(P_{j}^{l d}\right)$ is added to the actual power generation level.

Then, $P D_{j}^{v}$ is computed as:

$P D_{j}^{v}=P D_{j}+P_{j}^{l d}$

The transmission loss allocation (TLA) will carry out in the Stage 1, as:

\section{Stage 1: Transmission loss Allocation (TLA)}

In this stage, the power transmission losses are allocated to different systems buses. Then, both consumer and generation levels are modified from their physical levels to new virtual levels. The amount of TLA is either added to the load demand levels or subtracted from the power generation levels. In the first stage, three studied cases based on three TLA schemes which are: Z-bus TLA method [13], proportional rata based on load demand TLA method [13], and voltage based TLA scheme [12].

The MEBE are presented as:

1. If the total losses added to demand levels at different buses, then the MEBE is computed using the new virtual load demands as:

$$
G D_{i j}=P G_{i} P D_{j}^{v} / P_{d}^{s y s}
$$

The $P_{d}^{s y s}$ in this case equals to the total power generation or virtual load demand levels as:

$$
P_{d}^{s y s}=\sum_{i=1}^{N G} P G_{i}=\sum_{l=1}^{N D} P D_{j}^{v}
$$

2. If the total losses is subtracted from generation levels at different buses, then the new virtual power generation are used to compute the MEBE as: 


$$
G D_{i j}=P G_{i}^{v} P D_{j} / P_{d}^{s y s}
$$

The virtual power generation is subtracted the loss component certain bus from the power generation at this bus. The $P_{d}^{s y s}$ in this case equals to the total power generation/ virtual demand levels as:

$$
P_{d}^{s y s}=\sum_{i=1}^{N G} P G_{i}^{v}=\sum_{j=1}^{N D} P D_{j}
$$

\section{Stage 2: Allocation of the transmission usage costs}

The power flows in the transmission lines are computed using the MSF in (7). With the above decomposition, the effects of EBE on the power flow in line $\mathrm{k},\left(P F_{k}\right)$ is determined. The power flow in transmission line $k$ in terms of the EBE is computed as:

$$
P F_{k}=\sum_{i, j}\left(\left(D_{m}\right)_{k, i j} \cdot G D_{i j}\right)
$$

Under the MEBE principle, each flow component $\left(\left(D_{m}\right)_{k, i j} \cdot G D_{i j}\right)$, is deemed to "use" line $(k)$ irrespectively to its sign with respect to the net flow in line (k). Equation (27) presents the use of transmission line $\mathrm{k}$ by $\operatorname{EBE}\left(G D_{i j}\right)$. The usage of line $\mathrm{k}$ by demand at bus $\mathrm{j}$ is the sum of all MEBE, involved demand at bus $\mathrm{j}$, as:

$$
U D_{k j}=\sum_{i \in \Omega j}\left|\left(\left(D_{m}\right)_{k, i j} \cdot G D_{i j}\right)\right|
$$

The usage of line $\mathrm{k}$ by generator at bus $\mathrm{i}$ is the sum of all MEBE, involved generator at bus $i$, that is

$U G_{k i}=\sum_{j \in \Omega_{i}}\left|\left(D_{m}\right)_{k, i j} \cdot G D_{i j}\right|$

The total line k usage $\left(U L_{k}\right)$ due to all MEBE is:

$$
U L_{k}=\sum_{\substack{i \in \Omega_{j}, j \in \Omega_{i}}}\left|\left(D_{m}\right)_{k, i j} \cdot G D_{i j}\right|
$$

The transacted power rate in line $\mathrm{k},\left(r_{k}\right)$, can be computed from:

$$
r_{k}=C_{k} / U L_{k}
$$

\section{Scheme 8: Proposed MSF-Based TCA}

This scheme is based on the proposed MSF using Equation (6). A part of the power flow in line $\mathrm{k}$ is assigned directly to injection power at bus $\mathrm{i}$ as:

$$
P F_{k}^{i}=\left(D_{m}\right)_{k, i} . P I_{i}
$$

The usage of line $\mathrm{k}$ is computed from both sides $((U 1)$ and $(U 2))$ using the transacted power as:

$U 1_{k}^{i}=\left|\left(D_{m}^{(1)}\right)_{k, i} . P I_{i}\right|$ 
$U 2_{k}^{i}=\left|\left(D_{m}^{(2)}\right)_{k, i} \cdot P I_{i}\right|$

Where, $\left(D_{m}^{(1)}\right)_{k, i}$ and $\left(D_{m}^{(2)}\right)_{k, i}$ refer to the MSF of line $\mathrm{k}$ due to injection power at bus $\mathrm{i}$.

The maximum effective usage of line $\mathrm{k}, U e_{k}^{i}$, can be computed for minimum rates as:

$U e_{k}^{i}=\max \left(U 1_{k}^{i}, U 2_{k}^{i}\right)$

And, the minimum effective usage of transmission line $\mathrm{k}, U e_{k}^{i}$, can be computed for maximum transaction rates as:

$U e_{k}^{i}=\min \left(U 1_{k}^{i}, U 2_{k}^{i}\right)$

The transacted power rate in line $\mathrm{k},\left(r_{k}\right)$, can be expressed from:

$r_{k}=C_{k} / \sum_{i=1}^{N B} U e_{k}^{i}$

Where:

$C_{k}$ refers to the total annual cost of line k.

The cost allocated of each effective line usage is computed from:

$C_{k}^{i}=r_{k} \cdot U e_{k}^{i}$

\section{Applications:}

\subsection{Test systems}

The 5-bus test system [14] and IEEE 14-bus test systems [15] are used to show the capability of the suggested TCA schemes. The total annual costs of the transmission network are $1050 \$$ and $3627.64 \$$ for the two test systems, respectively. MATPOWER version 3.0 package [15] and MATLAB 6.5 Software are used to perform the suggested schemes.

\subsection{Results and Comments}

Tables 3 and 4 show different TCA schemes for the five bus test system. While, Tables 5 and 6 show different TCA schemes for the 14-bus test system. Tables 3 and 5 present the TCA for each bus due to network usage using suggested schemes (Schemes 1-6) for the two systems, respectively. Tables 4 and 6 present the TCA for each bus due to network usage for Schemes 7 and 8 compared with other two other conventional TCA methods namely, postage stamp method and Z-bus TCA method. Scheme 7 has three studied cases (Cases 1-3), while Scheme 8 has two studied cases (Cases 4 and 5).

\section{1) Five bus test system}

In Table 3 , Scheme 1 allocates the highest cost for the generation bus 1 which has the highest generation level (32.25\% of the total generation). While, Scheme 2 allocates the highest cost for the combined generator/load at bus 5 which has the highest loading level (40\% of the system load). In Scheme 3, the maximum TCA level is at bus 2 which equals $28.08 \%$ of the total network usage costs. The remaining schemes 
allocate varying TCA levels depending on the network topology, injected power at different buses and emphasis on current injection with some differences being relatively significant. Schemes 2 - 5 allocate the same TCA levels at buses 3 and 4 . But, in Schemes 1 and 6 the TCA levels at buses 3 and 4 aren't the same. These changes are due to the network topology. Both Schemes 1 and 6 allocate the highest TCA at Bus 2 due to the large number of lines connected to Bus 2. Due to the same reason, Schemes 1 and 6 allocate the lowest TCA levels at Bus 5 compared to other TCA schemes. In Table 4, the postage stamp and Z-bus TCA method allocate methods allocate different allocation levels compared to the suggested Schemes 1- 8. The Z-bus TCA method allocates the maximum TCA level at bus 1 (460.472 \$), which has the highest generation level and the minimum TCA level (41.49 \$) at Bus 5 which has the lowest power injection level. Scheme 7 is two-stage TCA scheme which has three studied cases. Case 1 allocates the transmission usage costs with the smallest level at Bus 2 which has the lowest load demand and the largest allocation level at Bus 5 which has the highest load demand level. While, Cases 2 and 3 allocate the transmission usage costs with the smallest level at Bus 5 and the largest level at Bus 1 (as similar to Z-bus TCA method). In Scheme 8, Cases 4 and 5 allocate the transmission usage costs with smallest allocation level at Bus 5 and the largest allocation level at Bus 1 . Similar to Z-bus TCA method, Schemes 7 and 8 allocate the same allocation levels at consumer Buses 3 and 4 similar to other schemes expect the Z-bus TCA method.

Table (3): Different proportional TCA schemes for 5-bus system (Schemes 1-6)

\begin{tabular}{|c|c|c|c|c|c|c|}
\hline Buses & Scheme 1 & Scheme 2 & Scheme 3 & Scheme 4 & Scheme 5 & Scheme 6 \\
\hline 1 & 341.25 & 117.6 & 209.26 & 233.72 & 231.9 & 160.02 \\
\hline 2 & 323.65 & 0 & 294.87 & 247.81 & 243.91 & 368.48 \\
\hline 3 & 136.34 & 259 & 151.63 & 127.43 & 129.5 & 186.43 \\
\hline 4 & 140.74 & 259 & 151.63 & 127.43 & 129.5 & 199.68 \\
\hline 5 & 108.02 & 414.4 & 242.61 & 313.61 & 315.19 & 135.39 \\
\hline Total \$ & 1050 & 1050 & 1050.01 & 1050 & 1050 & 1050.01 \\
\hline
\end{tabular}

Table (4): TCA results for 5-bus system (Schemes 7 and 8)

\begin{tabular}{|c|c|c|c|c|c|c|c|}
\hline \multirow{2}{*}{ Bus } & Postage Stamp & Z-bus & \multicolumn{3}{|c|}{ Scheme 7 } & \multicolumn{2}{c|}{ Scheme 8 } \\
\cline { 4 - 8 } & Method [4] & TCA [3] & Case 1 & Case 2 & Case 3 & Case 4 & Case 5 \\
\hline 1 & 301.82 & 460.472 & 120.643 & 383.241 & 383.214 & 383.214 & 372.669 \\
\hline 2 & 137.83 & 239.87 & 2.602 & 249.69 & 249.69 & 249.690 & 242.798 \\
\hline 3 & 131.25 & 153.63 & 259.001 & 198.757 & 198.757 & 198.757 & 207.747 \\
\hline 4 & 131.25 & 154.289 & 259.429 & 198.757 & 198.757 & 198.757 & 207.747 \\
\hline 5 & 347.83 & 41.49 & 408.325 & 19.582 & 19.582 & 19.582 & 19.038 \\
\hline Total (\$) & 1050 & 1050 & 1050 & 1050 & 1050 & 1050 & 1050 \\
\hline
\end{tabular}


Table (5): Different proportional TCA schemes for 14-bus system (Schemes 1-6)

\begin{tabular}{|c|c|c|c|c|c|c|}
\hline Bus & Scheme 1 & Scheme 2 & Scheme 3 & Scheme 4 & Scheme 5 & Scheme 6 \\
\hline 1 & 2068.8 & 0 & 1542.2 & 1480.1 & 1424.9 & 279.22 \\
\hline 2 & 863.65 & 303.94 & 420.88 & 550.02 & 540.85 & 296.77 \\
\hline 3 & 475.52 & 1319.4 & 660.78 & 634.17 & 659.7 & 258.88 \\
\hline 4 & 116.91 & 669.5 & 335.3 & 321.8 & 334.75 & 749.29 \\
\hline 5 & 3.066 & 106.45 & 53.312 & 51.165 & 53.224 & 144.52 \\
\hline 6 & 8.9901 & 156.87 & 78.564 & 75.401 & 78.435 & 529.56 \\
\hline 7,8 & 0 & 0 & 0 & 0 & 0 & 0 \\
\hline 9 & 13.10 & 208.68 & 104.50 & 100.29 & 104.39 & 0 \\
\hline 10 & 57.785 & 413.19 & 206.93 & 198.6 & 206.59 & 566.79 \\
\hline 11 & 5.8594 & 126.06 & 63.132 & 60.59 & 63.028 & 158.78 \\
\hline 12 & 0.78509 & 49.022 & 24.551 & 23.563 & 24.511 & 114.15 \\
\hline 13 & 2.0339 & 85.439 & 42.79 & 41.066 & 42.719 & 155.22 \\
\hline 14 & 11.15 & 189.09 & 94.698 & 90.885 & 94.543 & 374.46 \\
\hline Total $(\$)$ & 3627.65 & 3627.64 & 3627.64 & 3627.65 & 3627.64 & 3627.64 \\
\hline
\end{tabular}

Table (6): TCA results for 14-bus test system (schemes 7 and 8)

\begin{tabular}{|c|c|c|c|c|c|c|c|}
\hline \multirow{2}{*}{ Bus } & Postage Stamp & Z-bus & \multicolumn{3}{|c|}{ Scheme 7 } & \multicolumn{2}{c|}{ Scheme 8 } \\
\cline { 4 - 8 } & Method [4] & TCA [3] & Case 1 & Case 2 & Case 3 & Case 4 & Case 5 \\
\hline 1 & 1414.11 & 560.97 & 177.476 & 212.326 & 157.412 & 2808.46 & 2803.28 \\
\hline 2 & 551.67 & 1554.18 & 314.266 & 339.239 & 809.596 & 85.234 & 85.077 \\
\hline 3 & 659.70 & 488.88 & 1243.82 & 1221.094 & 1097.93 & 515.607 & 514.652 \\
\hline 4 & 334.76 & 138.98 & 629.484 & 619.623 & 480.174 & 132.762 & 132.517 \\
\hline 5 & 53.22 & 36.87 & 99.825 & 98.518 & $\mathbf{- 6 2 . 2 2 1}$ & 3.356 & 3.350 \\
\hline 6 & 78.44 & 126.63 & 147.087 & 145.183 & 286.609 & 7.289 & 7.275 \\
\hline 7,8 & 0 & 0 & 0 & 0 & 0 & 0 & 0 \\
\hline 9 & 206.59 & 303.03 & 389.377 & 382.405 & 458.620 & 45.631 & 50.473 \\
\hline 10 & 63.03 & 102.17 & 119.244 & 116.666 & 76.086 & 4.247 & 4.698 \\
\hline 11 & 24.51 & 33.99 & 46.271 & 45.370 & 26.577 & 0.642 & 0.710 \\
\hline 12 & 42.72 & 41.98 & 80.923 & 79.073 & 63.716 & 1.951 & 2.158 \\
\hline 13 & 94.54 & 120.09 & 179.792 & 174.997 & 100.889 & 9.556 & 10.570 \\
\hline 14 & 104.35 & 119.88 & 200.070 & 193.145 & 132.254 & 12.900 & 12.876 \\
\hline Total $(\$)$ & 3627.65 & 3627.64 & 3627.64 & 3627.640 & 3627.64 & 3627.64 & 3627.64 \\
\hline
\end{tabular}




\section{2) 14-bus test system}

In Table 5, Scheme 1 allocates the highest TCA level (2068.8 \$) for the generation Bus 1. While, Scheme 2 allocates the highest TCA level to the highest load demand at bus 3 (36.37\% of load demand). In Scheme 3, the maximum TCA level at bus 1 which has $42.51 \%$ of the total network costs. The remaining schemes allocate varying TCA levels depending on the network topology, injected power at different buses and emphasis on current injection with some differences being relatively significant.

In Table 6, the Z-bus TCA method allocate the maximum TCA at bus 2 which has the highest consumer level and the minimum TCA levels at Buses 7 and 8 whose have the minimum power injection levels. Scheme 7 is two-stage TCA procedure which has three studied cases. Cases 1 allocates the highest transmission usage costs, with TCA level 1243.82 \$, at Bus 3. Case 2 allocates the highest TCA level at Bus 3 (1221.094 \$). Also, Case 3 allocates the highest TCA level at Bus 3 (1097.93 \$).

In Scheme 8, Cases 4 and 5 allocate the transmission usage costs with the highest allocation levels at Bus 1 as $2808.46 \$$ and 2803.28 \$, respectively.

\section{Conclusions:}

In this paper, the problem of transmission cost allocation using different suggested schemes has been solved. Proportional rata TCA schemes are based on different ISO visions for both generation and load demand buses. The generalization the EBE for lossy networks has been effectively demonstrated. The MEBE based TCA scheme established the transmission individuals' cooperation and coordination. Another scheme has been suggested based on the modified sensitivity factors which are dependent on the actual system measurements. The TCA schemes are efficient, fair and equitable to participant agents.

Added to that, the suggested schemes are:

- Emphasizing the interaction among complex power and current associated with each network user and depending on the actual nodal currents, the exact network equations and circuit topology.

- Promoting more efficient utilization of generation and transmission resources.

- Defining and assigning the contributions of each generator/load to the transmission usage costs by system individuals.

- Helping and encouraging the participant to take an appropriate corrective action to reduce market risks. 


\section{References:}

[1] J. Pan, Y. Teklu, S. Rahman, and K. Jun, Review of Usage-Based Transmission Cost Allocation Methods under open access, IEEE Transactions Power System, Vol. 15, P. 1218-1223, 2000.

[2] F. D. Galiana, A. J. Conejo, and H. A. Gil, Transmission Network Cost Allocation Based on Equivalent Bilateral Exchanges, IEEE Trans. Power System, Vol. 18, P.1425-1431, 2003.

[3] A. J. Conejo, J. Contreras, D. A. Lima, and A. Padilha-Feltrin, Z-bus Transmission Network Cost Allocation, IEEE Transactions on Power Systems, Vol. 22, P. 342-349, Feb. 2007.

[4] J. Usaola, Transmission Cost Allocation in Pool Systems, the proceeding of $15^{\text {th }}$ PSCC, Session 40: Paper No. 1, 2005.

[5] J. M. Zolezz, and H. Rudnick, Transmission Cost Allocation by Cooperative Games and Coalition Formation, IEEE Transactions on Power Systems, Vol. 17, P. 1008-1015, 2002.

[6] A. Sedaghati, Cost of Transmission System Usage Based on an Economic Measure, IEEE Transactions Power System, Vol. 21, P. 466-473, 2006.

[7] P. A. Kattuman J. W. Bialek N. Abi-Samra, Electricity Tracing and Co-operative Game Theory, Proc. $13^{\text {th }}$ Power System Computation Conference, Trondheim, P. 238-243, 1999.

[8] D. Kirschen, R. Allan and G. Strbac, Contributions of Individual Generators to Loads and Flows, IEEE Transactions. Power Systems, Vol. 12, P. 52-60, Feb. 1997

[9] J. Bialek, Topological Generation and Load Distribution Factors for Supplement Charge Allocation in Transmission Open Access, IEEE Transactions on Power Systems, Vol. 12, P. 1185-1193, 1997.

[10] M. Pantoš et al., Modified Topological Generation and Load Distribution Factors, IEEE Transactions on Power Systems, Vol. 20, P. 1998-2005, 2005.

[11] Wai Y. Ng, Generalized Generation Distribution Factors for Power System Security Evaluations, IEEE Transactions PAS, Vol. 100, P1001-1005, $1981 .$.

[12] A. A. Abou El-Ela, and R. A. El-Sehiemy, Different Transmission Losses Allocation in Deregulated Power Markets, the proceeding of $42^{\text {nd }}$ International Universities Power Engineering Conference (UPEC 2007), Brighton, England, P. 634-640, Sept. 2007.

[13] A. J. Conejo, F. D. Galiana, and I. Kockar, Z-Bus Loss Allocation, IEEE Transactions on Power Systems, Vol. 16, P. 105-110, Feb. 2001.

[14] A. A. Abou El-Ela, M. Bishr, S. Allam and R. El-Sehiemy, Optimal Preventive Control Actions in Power System Using Multi-Objective Fuzzy Linear Programming Technique, Electric Power System Research Journal, Vol. 74, P. 147-155, April 2005. 
Proceedings of the $\boldsymbol{6}^{\text {th }}$ ICEENG Conference, 27-29 May, 2008

[15] R. D. Zimmerman, et al., MATPOWER Version 3.0, a MATLAB ${ }^{\mathrm{TM}}$ Power System Simulation Package, 2005, Available at: www.pserc.cornell.edu/matpower 